\title{
Voting Block Method for Verification of Beef and Pork using Back Propagation Learning Machines
}

\author{
Khoerul Anwar \\ Informatics Engineering STMIK \\ PPKIA Pradnya Paramita, \\ Indonesia
}

\author{
Sigit Setyowibowo \\ Informatics Engineering STMIK \\ PPKIA Pradnya Paramita, \\ Indonesia
}

\author{
Sujito \\ Informatics Engineering STMIK \\ PPKIA Pradnya Paramita, \\ Indonesia
}

\begin{abstract}
Identifying two digital image objects that have visually similar textures in the field of computer vision is a tough task. Usually the visual texture of an object has unique features from one another. However, the beef texture and pork texture both have similar visual textures. This visual similarity is often used by certain individuals to commit fraud in selling meat. It is important to conduct research on the texture similarity phenomenon in order to obtain a computational method that can be used to distinguish the two. So that this method can be applied in the field of computer vision. Until now, a mobilebased application for identifying beef and pork based on texture features in the community does not exist. In this condition, beef consumers often mistakenly recognize beef and pork. In this scientific paper, a beef and pork identification method is proposed based on the feature texture computation using the Vbloc method. The Vbloc (voting bloc) method is a classification technique that starts with partitioning the original image into $\mathrm{n}$ new image partitions (bloc). This bloc image is used as a testing image on ANN. It is different from the general classification where the training image and the testing image have the same dimensions. In the Vbloc method, the test image is cut into parts of a new image with smaller dimensions then the pieces are extracted. Next, testing is done on ANN to obtain the class from the input image. The method offered was tested on 61 pork images, 81 beef images and 10. The results obtained by the vblock method were able to identify the type of image correctly at $76.97 \%$.
\end{abstract}

\section{Keywords}

Voting block, identification, testure featurs, neural network, computer vision

\section{INTRODUCTION}

Identifying two digital image that have similar textures is a tough task in the field of computer vision. The visual texture of an object has a special characteristic. Therefore, features can be used as special features in the classification of an object. Features are very important to support performance in digital image identification [1] [2] [3].

Features are the unique characteristics of an image. Therefore, features are important in classifications involving large amounts of data with similar multi-objects [4]. Unique characteristics can be obtained by extracting the texture with the GLCM extraction technique [5]. Key features are an important part in supporting classification tasksi [6] [7]. The problem that is often faced is when two objects have similar feature values [8]. This visual similarity is often used by certain individuals to commit fraud in selling meat. So it is important to do research on the texture similarity phenomenon in order to obtain a computational method that can be used to distinguish the two. So that this method can be applied in the field of computer vision. Until now, there is no computer vision application based on mobile service to identify beef and pork in the community. This condition creates problems in the beef consumer community. Beef consumers often mistake fake beef.

Identifying beef and pork based on the feature value of the model class is the right step. The thing that is often faced is the identification of the test image against the model class is that high similarity elements are met in order to obtain good results. The basic identification problem is the availability of model classes. A class that is capable of adapting to new data, so that the system is able to generalize or predict new data with high accuracy. There are several classifiers that can be used to obtain a model class including: K-Mean [9], NN [22].

This paper propose a new model in identification, namely by using the Voting Block (VBloc) technique. The original image or the original image is cut into $\mathrm{n}$ parts, the image pieces are termed a block. Meanwhile, image extraction uses the GLCM method and feature selection uses the Minimum Overlaping Probability method anwar [10], The block image is used as the input image in the testing phase at ANN. The identification results are based on the highest results from the voting results of testing at ANN.

\section{RELATED WORK}

\subsection{Feature Extraction}

Beef and pork images that are indicated to have similar textures require a fairly detailed analysis of the features of each to distinguish between the two. There are several techniques that can be used for feature extraction including Wavelate [11] GLCM [12]. The beef and pork image has a homogeneous feature texture character. This means that there is no certain unique pattern found in the texture of the two. Therefore, in this study, a GLCM extractor was chosen to extract the image pixel characters at the grayscale level. In order to obtain features with a high enough difference between beef and pork, texture extraction is carried out with quite a lot of information from the extraction results obtained the value of beef and pork features. The value is then compared between beef and pork. Then a feature selection was made to get the GLCM features which had quite a big difference.

\subsection{Feature Selection}

The features used in this study adopt the results of Anwar's research [10] using the Minimum Overlap Probability (MOP) method. The two feature datasets that each contains 20 GLCM features from extraction results of beef and pork is likely to have four relationships, namely: independent, overlap (intersection), superset / subset and equal. In relation to these four relationships, in selecting feature, it is certainly targeted to get the strongest feature. The strongest feature is ideally an independent feature that does not have redundancy one another [13]. However, If beef and pork features do not have 
independent features, other solutions should be considered. In this paper, discussion is specified for features of two objects with overlap feature value. The method is used to select is Minimum Overlap Probability (MOP) [10]. MOP working principle is based on the overlap value of a feature from two different objects. The research objects are beef and pork digital images. 20 GLCM features for each digital image have dataset value. In each dataset, the minimum and maximum values for each feature can be determined. Based on the minimum and maximum values, it will be determined whether there is an independent feature or overlap occurs in feature's value.

MOP serves to select a feature with overlap value below the threshold. MOP checks the two datasets of features' values in beef and pork, and later calculate the feature overlap value. The range between the minimum and maximum values for a feature of a digital image of beef is symbolized by Fs, and for features of the digital image of pork is symbolized by F $b$. It can also be interpreted that $\mathrm{Fs}$ is a range of minimum point $\left(x_{1}\right)$ and maximum point $\left(x_{2}\right)$. Meanwhile, the $\mathrm{F} b$ length is $\mathrm{F} b$ between the maximum points $\left(x_{4}\right)$ and the minimum point $\left(x_{3}\right)$. From the description, the Fs area can be defined as follows:

$F s=x_{1}: x_{2}$

$$
\Delta x_{s}=\left|x_{2}-x_{1}\right|
$$

And $\mathrm{Fb}$ can be defined as follows:

$F b=x_{3}: x_{4}$

$$
\Delta x_{b}=\left|x_{4}-x_{3}\right|
$$

Fs and $\mathrm{Fb}$ have overlap values when meet the conditions

1. $\quad F s(\min )<\mathrm{Fb}(\min )$ and $\mathrm{Fs}(\max )>\mathrm{Fb}(\min )$ and $\mathrm{Fs}$ $(\max )<\mathrm{Fb}(\max )$

2. $\quad F s(\min )>\mathrm{Fb}(\min )$ and $\mathrm{Fs}(\min )<>\mathrm{Fb}(\max )$ and $\mathrm{Fs}$ $(\max )>\mathrm{Fb}(\max )$

Anwar [12] in his paper formulates the calculation range if two features have overlapped areas as follows:

$$
\text { L } o=\left|x_{2}-x_{13}\right| \text { or }\left|x_{4}-x_{1}\right|
$$

Referring to (3), then for the values of the features within may be indicated to yield problems to the identification process of digital images of cows and pigs. Problems may occur as in these areas there is a possibility of value duplication owned by cows and pigs features. The Lo value can be translated to be greater its value, thus the total duplication between pork and pork features is bigger and vice versa. What should be noted is the area is formed based on feature value range thus overlapped area of each feature is not absolute at certain degree as range is influenced by the stability of the minimum and maximum values for each feature. Nonetheless, by using area range based on the min-max feature, the system is still able to obtain the overlapped area. The possibility for overlap to happen or overlap probability becomes the major concern in this research. Furthermore, to determine the overlap probability of each feature between $F s$ and $F b$, it can be calculated by comparing Lo with overall feature's extent $(F S+F b)$. In this research, area overlap computation by the author is called overlap probability [10]. The formulation for computation is

$$
\text { Prob overlap }=L o /\left(\left(\Delta x_{s}+\Delta x_{b}\right)-L o\right)
$$

Equation (4) can be interpreted as; the smaller the Lo value, the smaller the probability overlap. Conversely, the bigger the Lo value, the greater the probability overlap value.

\section{MOP Algorithms}

1. Calculating min-max of extraction feature value of beef and pork digital images.
2. Determining the feature areas $\left(\mathrm{F}_{\mathrm{x}}\right)$ of beef and pork digital images.

a. If $F_{x}$ has no slices (disjoint) then it is the selected feature

b. if $F_{x}$ is a subset or superset of one another, then $\mathrm{F}_{\mathrm{x}}$ is unselected feature (rejected)

c. if in the delta Fx calculation of feature database is not included in the process to $a$ and $b$, then the process is continued (no 3 ).

3. Calculating the value of ProbError

4. Computing the ProbError value

5. Ranking

6. Determining the threshold (as selected feature selection filer)

7. Finding features with ProbError less than the threshold

\section{PROPOSED METHODE}

The author proposes this method because it is relevant for the case of image processing with a homogeneous texture. Bloc Voting is a calculation of the number of identities / labels of the bloc. The bloc identity is obtained from the classification of the bloc based on a predetermined model class. This method has two stages in the process, the first is creating a bloc image and the second is voting.

Voting is a voting mechanism based on majority votes. Voting is commonly used to find out how many members are affiliated in a group or class. Bloc is part of an area, in this study what is meant by bloc is part of an image (sub image). This means that the image is divided into $\mathrm{n}$ new images (bloc) with a smaller size and do not overlap with one another. The bloc image may have features that are identical to the parent image. The large number of bloc images that are identical to another image is suitable to say that these images are identical or have similarities. The classification stages with Vbloc are shown in Fig 1.

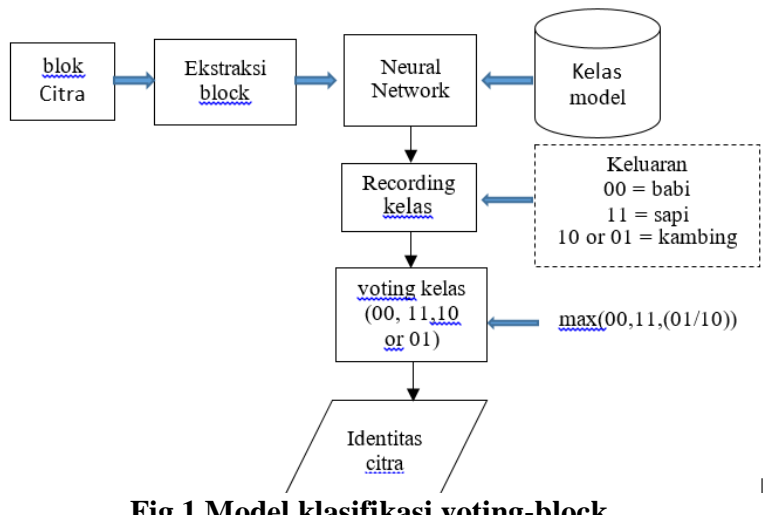

Fig.1 Model klasifikasi voting-block

\subsection{Bloc}

Bloc is a sub image of the original image. The size of the bloc can vary, but in this paper it is used in a certain size. The author offers a mathematical model in creating a bloc image from the original image. The original image is symbolized by Am x m which can be translated as an image with the size $\mathrm{m} \mathrm{x} \mathrm{m}$. Then the bloc image is a smaller image with the size $\mathrm{d} \mathrm{x} d$. The original image and the bloc image in this study are set as a square image. This equilateral image size makes it easy to create bloc and computations. In Fig 1, the original image and the bloc image with sizes are shown 


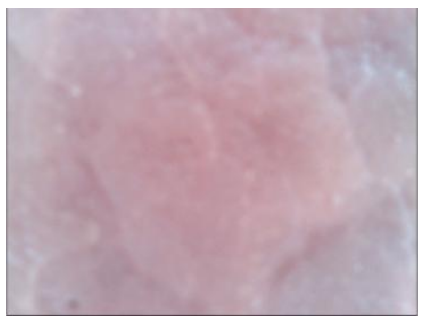

Original image $(255 \times 255)$

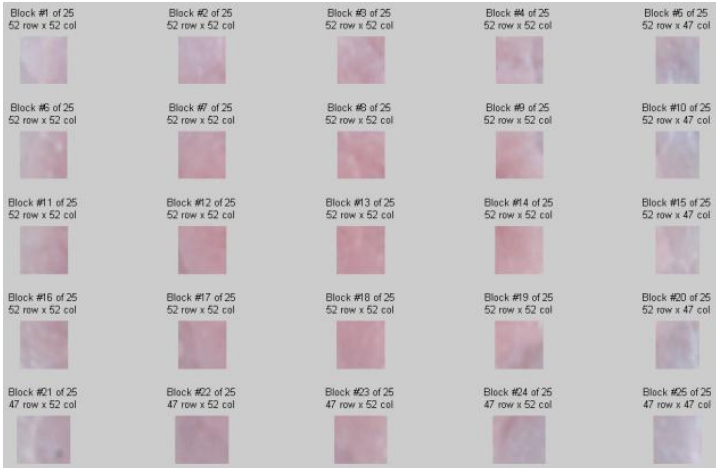

The resulting block image $(52 \times 52)$

Fig.2. Block Image

Determining the size and number of block images is done using a mathematical model as follows:

- Number of bloc images (p)

The number of blocks can be determined using a equation 4.1

$$
p=n^{2}
$$

$n$ is the number of blocks on the horizontal or vertical side of the original image window.

- $\quad$ block image size $(d)$

The size of the bloc image is obtained by dividing the length or width $(\mathrm{m})$ of the original size by the number of bloc (n), this is written as an equation 4.2

$$
d=m / n
$$

for the bloc image formed can be symbolized by $A_{P}^{d x d}, \mathrm{p}$ is the number of bloc and $d$ is the size of the bloc.

To illustrate the calculation to obtain the number and size of the bloc, an original image sample measuring 255 x $255(\mathrm{~m}=255)$ pixels using equation (5) and (6) obtained several block images as shown in Table 1.

Table 1. Block image size

\begin{tabular}{|c|c|c|}
\hline $\mathrm{n}$ & $\mathrm{p}=\mathrm{n}^{2}$ & $\mathrm{~d}=\mathrm{m} / \mathrm{n}$ \\
\hline 1 & 1 & $255 \times 255$ \\
\hline 2 & 4 & $128 \times 128$ \\
\hline 3 & 9 & $85 \times 85$ \\
\hline 4 & 16 & $64 \times 64$ \\
\hline 5 & 25 & $51 \times 51$ \\
\hline
\end{tabular}

\subsection{Ekstraksi Bloc}

At this stage, block (sub-image) extraction is based on the selected features using a GLCM extractor. Each block image $\left(A_{P}^{d x d}\right)$ is extracted based on the four selected features and a $\mathrm{p}$ value of feature extraction is generated. Then the extraction results are stored in a matrix with a size of $4 \times \mathrm{p}\left(M^{4 x p}\right)$. The $\mathrm{M}^{4}$ ${ }^{\mathrm{x} p}$ matrix becomes the input data for ANN for the classification process based on the Voting Bloc model.

The GLCM feature used for bloc extraction is the selected feature of the process using the Minimum Overlap Probability (MOP) method. The results obtained from the MOP application then selected four features with the highest MOP value. The selected features are Autocorrelation, information measure of correlation 2, Sum of square variance and Contrast.

\subsection{Block Classification}

\subsubsection{Block Testing}

The block classification stage is the process of classifying each block based on the target class that has been formed. A beef or pork image is inputted into the system and then the system is blocking it. The output of blocking is a number of new images. This new image is then extracted using the four selected features. Based on this extraction value, the system matches the model in ANN. The values for each block are stored in an array (Y). This data is used to vote on the testing output of the block. The algorithm for storing the test results of each block on the ANN is shown in Fig 3

$$
\begin{aligned}
& 1 \text { Setting counter } \mathrm{k}=\mathrm{p} ; \\
& 2 \text { while } \mathrm{k}<\mathrm{p} \\
& 3 \mathrm{Y}=\mathrm{JST}\left(\mathrm{net}, M^{4 x p}\right) \\
& 4 \mathrm{Y}(:, \mathrm{p})=\mathrm{Y} ; \\
& 5 \mathrm{k}=\mathrm{k}+1 ; \\
& 6 \text { end }
\end{aligned}
$$

\section{Fig.3 Block classification}

This algorithm describes the process of recording the classification results for each bloc into the three classes provided, namely pork, beef and non-pork cows. Storage of Y classification results $(:, p)$ based on each class of pigs, cows and non-pigs. Each Y output that has the value $[0 ; 0]$ is stored in the variable bb (pig) as data storage. Then the output that is valued at $[1 ; 1]$ is stored in the sp (cow) variable and both of them are stored in the nsp variable. In order the variables bb, sp and nsp are symbols of the pig, cow and non-cow_babi classes. Storage of each output into each class as shown in Fig 4.

$$
\begin{aligned}
& \text { 1. Start values } b b=0, s p=0, n s p=0 \\
& \text { 2. for } \mathrm{i}=1 \text { to } \mathrm{p} \\
& \text { 3. If } \mathrm{Y}(:, \mathrm{i})==[0 ; 0] \quad \mathrm{bb}=\mathrm{bb}+1 ; \\
& \text { 4. elseif } \mathrm{Y}(:, \mathrm{i})==[1 ; 1] \quad \mathrm{sp}=\mathrm{sp}+1 ; \\
& \text { 5. else } \mathrm{nsp}=\mathrm{nsp}+1 ; \\
& 6 \text { end } \\
& \text { 7. end }
\end{aligned}
$$

\section{Fig 4. Class member logging algorithm}

\subsubsection{Voting of the classification results}

The role of voting is to support the decision to determine the class of a test image in the predetermined target classes, namely pork, cattle and non-pork cows. Class determination is decided based on the voting results with the highest number of scores from each class. The voting is carried out using a formula 


$$
\text { Class identity }=\max (\mathrm{bb}, \mathrm{sp}, \mathrm{nsp})
$$

The description of bb is the image of pork, sp is the image of beef and nsp is the image of meat, not both. By using (7), it can be seen the results $A^{m \times n}$ of the test image voting. Illustration in formula 4.3, if it is known that an image of a beef is done the block process becomes 16 new images. The new image is tested on ANN and produces data $\mathrm{bb}=4, \mathrm{sp}=12, \mathrm{nsp}=0$. Then the winner of the voting result is the beef image $(\mathrm{sp}=12)$. So that the beef image is recognized as a beef image based on the results of the test voting on ANN.

\section{EXPERIMENT AND RESULTS}

Experiments were carried out by blocking images of various sizes. Block variations as shown in Table 1. Each of these variations is tested on ANN. Fig 5. Shown the correlation of block size with the results of model accuracy. The accuracy of the vbloc method decreases as the block image size gets smaller. This means that the more block images or image pieces with a smaller size, the block image tends to be wrongly used as an identification technique. The block variation with a size that is much smaller than the original size results in a smaller accuracy value. A more detailed analysis of the classification output obtained two anomalies, namely: 1) the accuracy of the vblock method decreased, but then it stabilized when the block was $49 \times 65$ to $32 \times 43.2$ ) In this stable condition, it was found that the beef was correctly identified (true positive) entirely, meanwhile pork is identified as false (false positive) entirely. The two anomalies are caused by: 1) the smaller the image block size has the extraction feature value greater than the original image, 2) the feature value in the block image for beef is greater than the beef training data value so that the beef block image is identified correctly all $(100 \%)$. Likewise, the feature value of the pork block image is greater than the pork training data and is within the range of the beef feature value so that the pork block image is all identified incorrectly $(0 \%)$ or is recognized as whole beef.

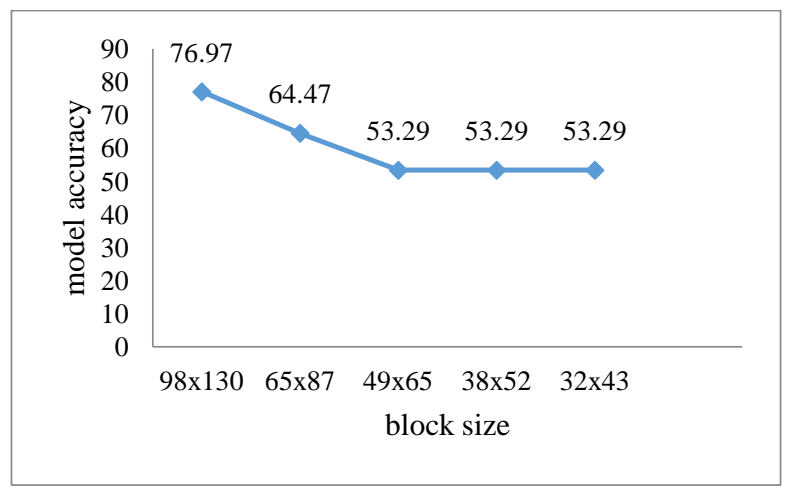

Fig 5. Effect of block size on accuracy

Model analysis using confusion matrix against the vblock model with the best model performance at the size of $98 \times 130$, the results are as shown in Table 2.

Table 2 Confusion matrix vblock

\begin{tabular}{c|ccccc}
\hline \multicolumn{5}{c}{ Identified } \\
Real & Meat & Pork & Beef & Others & $\Sigma$ \\
& Pork & 39 & 13 & 9 & 61 \\
& Beef & 0 & 78 & 3 & 81 \\
& Other & 0 & 10 & 0 & 10 \\
& $\Sigma$ & 39 & 101 & 12 & 152 \\
\hline
\end{tabular}

The comparison of the classification results of the vbloc method with other methods is shown in Table 3.

Table 3. The performance of the proposed method

\begin{tabular}{|c|c|c|c|c|c|}
\hline $\begin{array}{c}\text { N } \\
\mathbf{0}\end{array}$ & \multicolumn{1}{c}{$\begin{array}{c}\text { Metho } \\
\text { de }\end{array}$} & \multicolumn{1}{c|}{$\begin{array}{c}\text { Accu } \\
\text { racy } \\
(\mathbf{\%})\end{array}$} & $\begin{array}{c}\text { Precis } \\
\text { ion } \\
(\boldsymbol{\%})\end{array}$ & $\begin{array}{c}\text { Sensitivit } \\
\mathbf{y} \\
(\boldsymbol{\%})\end{array}$ & $\begin{array}{c}\text { Specifici } \\
\text { ty (\%) }\end{array}$ \\
\hline $\mathbf{1}$ & $\begin{array}{c}\text { MOP- } \\
\text { BPNN }\end{array}$ & 90,79 & 90,85 & 93.48 & 64,29 \\
\hline $\mathbf{2}$ & $\begin{array}{c}\text { Pro } \\
\text { posed } \\
\text { method }\end{array}$ & 76,97 & 82,39 & 99,15 & 0.00 \\
\hline
\end{tabular}

\section{CONCLUSSIONS}

The proposed vblock method can be used to identify beef and pork with an accuracy rate of $76.97 \%$ with an image size of 98 x 130 pixels. The vblock method results in an analysis that the smaller the block size, the lower the identification accuracy.

For developing this work, authors suggest some future works as explained: It is possible to use other metrics with cohesion and coupling metrics for computing reusability such as : complexity, flexibility metrics

\section{REFERENCES}

[1] M. P. a. B. M.-I. Eva Alica Tzschaschel, "The Effect of Texture on Face Identification and Configural Information Processing," PSIHOLOGIJA, vol. 47, no. 4, pp. 433-447, 2014.

[2] S. F.-E. Laleh Armi, "Texture Image Analysis and Texture Classification Methods a Review," International Online Journal of Image Processing and Pattern Recognition, vol. 2, no. 1, pp. 1-29, 2019.

[3] S. S. M. Candra Dewi, "Texture Feature On Determining Quantity of Soil Organic Matter For Patchouli Plant Using Backpropagation Neural Network," Journal of Information Technology and Computer Science, vol. 4, no. 1, pp. 1-14, 2019.

[4] V. R. N. M. S. Manikandan, "Analysis of Ultra Sound Kidney Image Features for Image Retrieval by Gray Level Co-Occurrence Matrices," Lecture Notes on Software Engineering, Vol. 1, No. 1, February 2013, vol. 1, no. 1, pp. 94-97, 2013.

[5] Z. H. M. S. L. M. N. T. Mahfuzah Mustafa, "GLCM Texture Feature Reduction for EEG Spectrogram Image using PCA," in Proceedings of 2010 IEEE Student Conference on Research and Development (SCOReD 2010),, Putrajaya, Malaysia, 2010.

[6] R.-G. W.-M. W. W. G. Yang Zhao, "Local Quantization Code Histogram For Texture Classification," Neurocomputing, vol. 207, pp. 354-364, 2016.

[7] R.-G. W. W.-M. W. G. Yang Zhao, "Local Quantization Code histogram for texture classi?cation," Neurocomputing, vol. 207, pp. 354-364, 2016.

[8] N. A. V. M.-W. S. A. Abdullah Iqbal, "Classi?cation of pre-sliced pork and Turkey ham qualities based on image colour and textural features and their relationships with consumer responses," Meat Science, vol. 84, p. 455-465, 2010.

[9] S. P. M. Dewi Pramudi Ismi, "K-means clustering based filter feature selection on high dimensional data," International Journal of Advances in Intelligent 
Informatics, vol. 2, no. 1, pp. 38-45, 2016.

[10] K. Anwar, A. Harjoko and Suharto, "Feature Selection Based on Minimum Overlap Probability (MOP) in Identifying Beef and Pork," (IJACSA) International Journal of Advanced Computer Science and Applications, vol. 7, no. 3, pp. 316-322, 2016.

[11] Z. X. Zhang Hong, "Texture feature extraction based on wavelet transform," in International Conference on Computer Application and System Modeling (ICCASM 2010), 2010.

[12] O. B. Abouelatta, "Classification of Copper Alloys Microstructure using Image Processing and Neural Network," Journal of American Science, vol. 9, no. 6, pp. 215-223, 2013.
[13] S. Y. Cho and H. R. Byun, "Human activity recognition using overlapping multi-feature descriptor," ELECTRONICS LETTERS, vol. 47, no. 23, pp. 1275 1277,2011

[14] A. Mathur and G. M. Foody, "Multiclass and Binary SVM Classi?cation: Implications for Training and Classi?cation Users," IEEE GEOSCIENCE AND REMOTE SENSING LETTERS, vol. 5, no. 2, pp. 241-245, 2008.

[15] T. Wakahara and Y. Yamashita, "k-NN classi?cation of handwritten characters via accelerated GAT correlation," Pattern Recognition, vol. 47, p. 994-1001, 2014.

[16] EnginEsme and B. Karlik, "Fuzzy c-means based support vector machines classifier for perfume recognition," Applied Soft Computing, vol. 46, p. 452-458, 2016. 